\title{
LOS NUEVOS SISTEMAS INTELIGENTES DE TRANSPORTE PARA UNA CIUDAD EN DESARROLLO, LIMA PERÚ
}

\section{THE NEW ITS SERVICES FOR A DEVELOPING CITY, LIMA, PERU}

\author{
Manuel J. Martínez
}

\section{RESUMEN}

Este artículo presenta ocho Sistemas Inteligentes de Transporte adecuados a una ciudad en desarrollo en un proceso de motorización, dirigidas principalmente a mejorar el atractivo del transporte público en relación a autos y taxis, y así reducir la congestión y la contaminación sin costo social, utilizando para ello sistemas avanzados de telecomunicaciones para obtener información en tiempo real de tráfico vehicular y así elevar la calidad de operaciones y control de tráfico y de transferencias intermodales.

Palabras clave: Transporte, Tren, Ciudad, Telecomunicaciones

\begin{abstract}
Keywords: Transportation, City, Rail, Transit, Telecommunications

\section{A. LOS BENEFICIOS DE LOS SISTEMAS INTELIGENTES DE TRANSPORTE (SIT)}

This paper presents eight services of Intelligent Transportation Systems modified to fit a developing city in a motorization process, to improve transit attractiveness over auto and taxi, and thus reducing congestion and pollution.

transporte de buses rápidos confronta escalamientos de costos de construcción, y al mismo tiempo, exorbitantes
Los Sistemas Inteligentes de Transporte (SIT) se basan sobre la información de tráfico vehicular en tiempo-real ${ }^{1}$. Los SIT mejoran la eficiencia y la productividad en el uso de la infraestructura de transporte al distribuir la demanda más equitativamente sobre la capacidad de las vías de transporte, al reducir la congestión causada por incidentes imprevistos, y al reducir el consumo de combustible al distribuir el tráfico a vías menos congestionadas [1]. En el caso de Lima, los beneficios de los SIT pueden incrementarse considerablemente si se dirigieran a transferir la demanda de transporte de menor productividad (autos y taxis) hacia el modo de transporte de mayor productividad (Tren Urbano)

\section{B. LOS SIT EN CIUDADES EN DESARROLLO}

Actualmente, los países en desarrollo tienen la oportunidad de implementar servicios SIT de bajo costo que ya han sido comprobados en países desarrollados e impulsados por el desarrollo de los teléfonos celulares y de la Internet. Esta oportunidad es más atractiva cuando el crecimiento urbano es explosivo mientras que el financiamiento para infraestructura de transporte es muy escaso. Así, se han recomendado políticas de implementación acelerada de SIT desde diversos organismos incluyendo el Banco Mundial [2]

En Lima, un factor determinante a favor de los servicios SIT es que, los proyectos convencionales de transporte están encontrando desafíos inesperados de implementación, que todavía no pueden superar. Las ciclovías son inefectivas para atraer usuarios, y el costos sociales al competir por la muy escasa infraestructura de transporte contra otros operarios de transporte público y al tener que construir en túnel [3]. Por eso, al buscarse alta efectividad, se diseñan estos SIT asociados al Tren Urbano, pues no compite por infraestructura contra otros modos de transporte.

\section{LOS SERVICIOS SIT CONVENCIONALES}

Los servicios SIT convencionales que tomamos en cuenta son los siguientes:

- Sistema avanzado de información de tráfico en tiemporeal [4]

- Sistema avanzado de operaciones y control de tráfico en tiempo-real $[4,5]$

- Guía e información en tiempo real de estacionamiento de autos [6]

- Información en tiempo-real de índices de contaminación [7]

- Información en tiempo-real del transporte público [8]

- Operaciones y control de tráfico que favorecen al transporte público [8]

- Letreros luminosos que anuncian información de tráfico en tiempo-real [9]

- Comunicaciones dinámicas de corto alcance (CDCA ${ }^{2}$ [10]

- Coordinaciones en tiempo-real entre líneas de transporte público [11]

- Monitoreo de velocidad del transporte público usando vehículos probe [12]

- Asignación de uso de carriles usando letreros luminosos $\quad y \quad$ semáforos [13]

\footnotetext{
a Doctor of Philosophy in Transportation (Major in Transportation Engineering) por la New Jersey Institute of Technology. Humphrey Fellow especializado en Urban Transportation Planning and Policy por la Rutgers, The State University of New Jersey. Economista por la Pontificia Universidad Católica del Perú, Registro del Colegio de Economistas de Lima 4047.

1 Tiempo-real significa: en forma simultánea al momento en que se producen los hechos.

${ }^{2}$ En inglés DSRC, dynamic short range communications, el cual puede usarse para ubicar e identificar vehículos a corta distancia a la entrada de instalaciones ó peajes. 
- Software de coordinación de usuarios de autos particulares (carpool) [14]

\section{EL ÍNDICE DE RENDIMIENTO}

La efectividad de las vías de transporte de una ciudad en desarrollo, se mide como una extensión de la efectividad del transporte público por ser éste el transporte de uso mayoritario. El punto de partida es el concepto de Productividad de Ruta [15]. La fórmula de espacios-km/h se modifica reemplazando espacios por personas, personas-km/h y personas-km/h-carril. Se le nombra el Índice AATE $^{3}$ de productividad de transporte.

\section{LOS NUEVOS SERVICIOS SIT}

Los nuevos servicios SIT se obtienen al modificar las piezas del capítulo $\mathrm{C}$ :

Centro Avanzado de Mercadeo e Información SurcoAviación CAMISA. El centro CAMISA consiste en una oficina que centralizará la información proveniente de detectores de tráfico, la procesará y convertirá en los servicios SIT, los cuales se comunicarán a los usuarios. Tiene el objetivo de promocionar y facilitar la transferencia de pasajeros desde los modos de transporte autos y taxis al Tren Urbano.

\section{Servicio 1. Información de tráfico vehicular en tiempo-real impartida a autos $y$ taxis en comparación al Tren Urbano. Este servicio distribuye información del tráfico en tiempo-real a autos y taxis para promover al Tren Urbano como alternativas al automóvil. Este servicio incluye la recolección de datos de tráfico vehicular, usándose para ello uno o varios de los tipos de sensores existentes: loop-detectors, video image processing, microwave sensors, acoustic sensors, etc., los cuales aún necesitan de un análisis para determinar los más adecuados para Lima. Luego, se transmiten los datos al centro CAMISA donde se integran con información del Tren Urbano, se procesa y se transmite a los usuarios de auto y taxis por medio de ipod, radio, teléfonos celulares y otros medios a seleccionarse posteriormente. Se espera que el Tren Urbano ofrezca un servicio más rápido en relación al auto, al acentuarse el proceso de motorización sobre una reducida capacidad vial. También se espera que este servicio sea efectivo porque al ser Lima una ciudad compacta, varios orígenes y destinos de los usuarios actuales de autos y taxis serán servidos por el Tren Urbano}

Servicio 2. Información comparativa en tiempo-real del Tren Urbano a pasajeros y operarios de transporte público. Se imparte la información del tráfico en tiemporeal a operarios y a pasajeros del transporte público. Este servicio requiere de un sistema de detección de velocidad de transporte público y de un receptor de la información de tipo teléfono celular. Al conocerse la velocidad comparativa entre transporte público y Tren Urbano así como la cantidad de personas que están esperando en paraderos de las estaciones del Tren urbano, el efecto natural será un reordenamiento del transporte público de acuerdo al flujo de pasajeros desde/hacia estaciones del Tren Urbano, sin necesidad de eliminar puestos de trabajo del transporte público

Servicio 3. Control avanzado de las instalaciones de transferencia de las estaciones del Tren Urbano. Este servicio facilita las transferencias de auto, taxi, y transporte público a Tren Urbano, mediante el control de carriles especiales desde las inmediaciones de la estación del Tren Urbano hasta las áreas de embarque/desembarque, así como a la playa de estacionamiento. La identificación de vehículos será posible por medio de la tecnología CDCA. Este sistema se complementa con cámaras de videos de seguridad, conteo automático de pasajeros en espera, usos de semáforos, detectores de tráfico de precisión y señalizaciones usando letreros luminosos, y otros. En este caso los sensores tendrán el objetivo de reconocimiento de vehículos por tipo y por tamaño, y también del control del tiempo de atención del transporte público, autos y taxis en las áreas de embarque y desembarque

Servicio 4. Maximización de la productividad de vías saturadas de acceso al Tren Urbano. Este servicio tiene el objetivo de distribuir la capacidad vial entre diversas clases de tráfico de tal manera de maximizar la productividad de la vía de transporte. La medida de productividad del transporte a utilizarse es el Índice AATE. Este sistema detecta el número de personas como parámetro para distribuir la capacidad vial entre diversas clases de tráfico, una situación propia de las calles de Lima. Se aplica en las áreas de influencia del Tren Urbano. Luego de medir el Índice AATE, se calculan las posibles mejoras derivadas de operaciones y control de tráfico, considerándose entre éstas los carriles reversibles, asignación de "luz verde", preferencia por algún tipo de vehículos, transbordo entre vehículos de transporte público, etc. Esta información está dirigida a funcionarios municipales y policiales que tomen decisiones en tiempo-real en campo de operaciones. Este sistema aliviará la congestión y, en su efecto secundario, mejorará el acceso a las estaciones del Tren Urbano

Servicio 5. Monitoreo avanzado en tiempo-real de emisiones contaminantes del transporte urbano. Tiene el objetivo de medir la contaminación sobre la infraestructura de transporte, con fines de alertar a los usuarios y además proveer de un índice complementario para operaciones y control de tráfico. Este servicio consta del despliegue de sensores de la calidad del aire en las vías de transporte en el área de influencia del Tren urbano. Esta información es procesada en el centro CAMISA con los modelos matemáticos de transporte y de tráfico a partir de los cuales se obtienen los índices para usuarios por zonas. Entre los usuarios se cuentan las autoridades ambientales, municipales, y policiales.

Servicio 6. Detección avanzada de velocidad en tiemporeal usando transporte público. Este servicio tiene el objetivo de recolectar información de velocidad de transporte público y de autos con el fin de procesarla y servir de insumo a los demás servicios SIT. Consiste en el uso de teléfonos celulares con GPS implantados en los vehículos de transporte público y con capacidad de transmisión de los datos al centro CAMISA, donde se les procesa para calcular las velocidades. Los mismos teléfonos celulares podrán luego recibir información de los demás servicios SIT (por ejemplo Servicio 2) 
Servicio 7. Coordinación avanzada taxi-tren. El servicio taxi-tren consiste en un servicio de tipo "colectivo" para transportar a personas desde origen (tal como domicilio) a estación de tren y desde estación de tren a destino (tal como centro de trabajo). Este servicio consta de un servicio automático de coordinación en tiempo-real, cuyo procesamiento se realiza en centro CAMISA. Así, el taxi recibe un rol de pasajeros que recoger y una ruta óptima para recorrer los orígenes previamente registrados en una base de datos digital georeferenciada con restricciones de horario, y luego vuelve a hacer el servicio desde estación de Tren Urbano, pero esta vez el taxi distribuye a las personas en tiempo y ruta óptima, usando una ruta óptima de destinos. El sistema se activa con una tarjeta magnética pre-pago que incluye los datos de origen, destino, y horarios del usuario. Esta información y los de los demás pasajeros del taxi se transmiten a centro CAMISA vía teléfono celular (ó por Internet) donde se procede a elaborar en tiempo real la ruta óptima, la cual se presenta al taxista en forma de mapa con direcciones. El efecto será el de un servicio taxicolectivo de alta calidad y eficiencia

Servicio 8. Información avanzada de estacionamiento y reserva de espacio. Este servicio tiene el objetivo de usar la capacidad de estacionamiento de las estaciones de Tren Urbano y así brindar un servicio sin interrupciones al automovilista que transfiere a Tren Urbano así como servicios de limpieza y seguridad. El servicio consiste en información en tiempo-real

acerca de la existencia de espacios disponibles y ubicación de los mismos, así como también la reserva anticipada de los espacios por medio de teléfonos celulares, Internet, y otros canales. Este sistema se compone de sensores de entrada y salida de la playa de estacionamiento, y de sensores de presencia de vehículos en los espacios del estacionamiento, así como sistema de señales y semáforos para guiar al automovilista a encontrar el espacio requerido. El control se realizará desde cada estación de Tren Urbano aunque con información centralizada en centro CAMISA

\section{CONCLUSIONES Y RECOMENDACIONES}

- Se han obtenido nuevos y originales servicios SIT, al modificar un conjunto de servicios SIT convencionales para adecuarlos a Lima, de tal manera de variar un aspecto de los originales SIT; un aspecto que puede ser el uso, ó capacidades técnicas, ó su alcance geográfico, ó la medición de una variable especial para Lima, etc.

- Estos nuevos servicios SIT aprovechan oportunidades que surgen de la situación de Lima: a) maximizar la transferencia de pasajeros de autos y taxis a Tren Urbano, b) maximizar la productividad de las vías de transporte por tipo y tamaño de vehículo, c) facilitar que el transporte público se pueda adecuar a la demanda de pasajeros de/hacia estaciones de Tren Urbano sin necesidad de eliminar empleos, d) aprovechar la gran cantidad de taxistas que podrían actuar en los accesos a estaciones de Tren Urbano en vez de congestionar las vías en las horas punta, y e) aprovechar que Lima es una ciudad compacta y que varios orígenes y destinos de usuarios de autos y taxis serán servidos por el Tren Urbano

- El centro avanzado CAMISA (centro avanzado de mercadeo e información Surco-Aviación) recolecta la información de los sensores de las vías de transporte y del Tren Urbano, la procesa, y la imparte a usuarios

- Este artículo presenta una artillería de ocho servicios SIT, diseñados para aprovechar las oportunidades de Lima y así obtener alta efectividad en promover las transferencias de usuarios de autos y taxis a Tren Urbano

- Se recomienda calcular el mercado potencial de los usuarios de los ocho servicios SIT de tal manera de verificar la cantidad de usuarios de auto y taxi que tengan origen y destino sobre el área de influencia del Tren urbano así como el nivel de congestión y su efecto sobre los usuarios

- Se recomienda calcular los beneficios alcanzables por los nuevos servicios SIT en sus efectos sobre la contaminación y la congestión de Lima

- Se recomienda elaborar la ingeniería básica de los servicios SIT, con especial énfasis en seleccionar las tecnologías adecuadas de sensores de tráfico vehicular, de las capacidades y funciones del centro CAMISA, y de transmisión de datos a usuarios

\section{AGRADECIMIENTOS}

EI CONCYTEC, Consejo Nacional de Ciencia y Tecnología financia el Contrato 194-2005-CONCYTEC-OAJ (manuscrito a mayo 2006. 


\section{REFERENCIAS BIBLIOGRAFICAS}

1. [ITS America Benefits, Evaluation, and Costs Committee. 1994. Proceedings of the Workshop "Assessment of the Benefits and Costs of the ITS Services". Newport. CA

2. [Yokota, Toshiyuki, Haruo Ishida, and Kotaro Kato. 2004. "Deployment of Intelligent Transportation Systems in Countries with Developing and Traditional Economies. ITS Toolkit". IN Transportation Research Record: Journal of the Transportation Research Board, No 1886. TRB, National Research Council, Washington, D.C. pp:1-9

3. Martinez, Manuel J. 2007. "Challenges to Conventional Transportation Projects and the Potential of Intelligent Transportation Systems in a Developing City, Lima, Peru". EN Revista ECIPerú 2006-2007 Vol. 3 No. 2 pp:2-8. Lima

4. New Jersey Institute of Technology. 1994. Intelligent Vehicle Highway Systems: Why, What and How?. Proceedings of the FHWA seminar on IVHS Functional Requirements and Technologies. Newark, New Jersey

5. Johnson, Christine M., and Edward L. Thomas. 1999. Transportation Management Center Concepts of Operation Implementation Guide. USDOT. Washington, DC

6. Shaheen, Susan A. And Caroline Rodier. 2007. "Smart Parking management to Boost Transit, Ease Congestion. Oakland, California, Field Test Shows Promise". IN TR News July-August Number 251 pp:30-31. Transportation Research Board of the National Academies. Washington, DC

7. LaBreche, Timothy M.C., Robert B. Jacko, Barry K. Partridge, William Schneider, and Ahmed Soliman. 2002. Effectiveness of the Borman Expressway ITS Program on Local Air Quality. Final Report. Joint Transportation Research Program. Purdue University. Sponsor Indiana DOT

8. Yim, Youngbin, and Jean-Luc Ygnace. 1993. Observations on European Advanced Traveler Information and Traffic Management Systems. California PATH Program. ITS. University of California, Berkeley

9. APTA. 2006. "ROMANSE Aims to Reduce Auto Dependency". IN International Focus 06/30/06 http://www.apta.com/intnatl/intfocus/romanse2.cfm

10. Kim, Jeongho, and Kyungwon Lee. 2001. "A Study on the ITS-BIS Service and Traffic Management using DSRC Protocol". IN CDROM of Proceedings of $8^{\text {th }}$ ITS World Congress. Sydney Australia

11. Miller, Mark A., Dimitri Loukakos. 2001. "How ITS Can Promote Coordinated Passenger Intermodal Operations and Services: An Institutional Perspective". IN CDROM of Procs. of $8^{\text {th }}$ ITS World Congress. Sydney Australia

12. Yi, Ping, Haiyan Zhen, and Yucheng Zhang. 2004. "Assessment of Traffic Volume, Based on Travel Time, to Enhance Urban Network Operation". IN TRR: Journal of the Transportation Research Board No. 1878 TRB, NCR, Washington, D.C. pp:164-170

13. Daganzo, Carlos F., Jorge Laval, and Juan Carlos Muñoz. 2002. Ten Strategies for Freeway Congestion Mitigation with Advanced Technologies. California PATH Program. ITS. University of California, Berkeley

14. Kocur, G., D. Zaelke, and L. Neumann. 1977. Feasibility Study of Shared Ride Auto Transit: Final Report. Report UMTA IT 06-0144-77-1. Service and Demonstration Program. USMTA-USDOT

15. Vuchic, Vukan R. 1981. Urban Public Transportation. Systems and Technology. Prentice-Hall, Inc. Englewood Cliffs, New Jersey 07632

E-mail: mjmartinez@pucp.edu.pe 